\title{
Multisite versus multiorbital Coulomb correlations studied within finite-temperature exact diagonalization dynamical mean-field theory
}

\author{
A. Liebsch, ${ }^{1}$ H. Ishida, ${ }^{2}$ and J. Merino ${ }^{3}$ \\ ${ }^{1}$ Institut für Festkörperforschung, Forschungszentrum Jülich, 52425 Jülich, Germany \\ ${ }^{2}$ College of Humanities and Sciences, Nihon University, Tokyo 156, Japan \\ ${ }^{3}$ Departamento de Física Teórica de la Materia Condensada, Universidad Autónoma de Madrid, Madrid 28049, Spain
}

(Received 16 September 2008; published 28 October 2008)

\begin{abstract}
The influence of short-range Coulomb correlations on the Mott transition in the single-band Hubbard model at half filling is studied within cellular dynamical mean-field theory for square and triangular lattices. Finitetemperature exact diagonalization is used to investigate correlations within two-, three-, and four-site clusters. Transforming the nonlocal self-energy from a site basis to a molecular-orbital basis, we focus on the interorbital charge transfer between these cluster molecular orbitals in the vicinity of the Mott transition. In all cases studied, the charge transfer is found to be small, indicating weak Coulomb-induced orbital polarization despite sizable level splitting between orbitals. These results demonstrate that all cluster molecular orbitals take part in the Mott transition and that the insulating gap opens simultaneously across the entire Fermi surface. Thus, at half filling we do not find orbital-selective Mott transitions or a combination of band filling and Mott transition in different orbitals. Nevertheless, the approach toward the transition differs greatly between cluster orbitals, giving rise to a pronounced momentum variation along the Fermi surface, in agreement with previous works. The near absence of Coulomb-induced orbital polarization in these clusters differs qualitatively from single-site multiorbital studies of several transition-metal oxides, where the Mott phase exhibits nearly complete orbital polarization as a result of a correlation driven enhancement of the crystal-field splitting. The strong singleparticle coupling among cluster orbitals in the single-band case is identified as the source of this difference.
\end{abstract}

DOI: 10.1103/PhysRevB.78.165123

PACS number(s): 71.18.+y, 71.27.+a

\section{INTRODUCTION}

Considerable progress has recently been achieved in the understanding of the Mott transition in a variety of transition-metal oxides. ${ }^{1}$ Whereas density functional theory in the local density approximation (LDA) predicts many of these materials to be metallic, the explicit treatment of local Coulomb interactions via dynamical mean-field theory (DMFT) (Ref. 2) correctly yields insulating behavior for realistic values of the on-site Coulomb energy $U$. In the metallic phase, the noncubic structure of some of these systems gives rise to nonequivalent, partially filled subbands that are split by a crystal field and exhibit orbital-dependent electron occupancies. The hallmark of the Mott transition of these oxides is that orbital polarization can be greatly increased by Coulomb correlations and that the insulating phase is nearly completely orbitally polarized. For instance, in the case of $\mathrm{LaTiO}_{3}$, the $e_{g}^{\prime}$ bands are pushed above the Fermi level and the remaining singly-occupied $a_{g}$ subband is split into lower and upper Hubbard bands. ${ }^{3,4}$ In the case of $\mathrm{V}_{2} \mathrm{O}_{3}$, Coulomb correlations push the $a_{g}$ band above the Fermi level, and the doubly-degenerate $e_{g}^{\prime}$ subbands exhibit a Mott gap. ${ }^{5,6} \mathrm{Also}$, in the insulating phase of $\mathrm{Ca}_{2} \mathrm{RuO}_{4}$, the $d_{x y}$-like band is completely filled, and the $d_{x z, y z}$-like subbands are split into Hubbard bands. ${ }^{7,8}$ The common feature of the Mott transition in these materials is that the effective band degeneracy is reduced from three to two or one so that the critical Coulomb energy is lower than it would be if the $t_{2 g}$ bands were fully degenerate. On the other hand, other materials can exhibit a quite different behavior. For instance, orbital polarization in $\mathrm{BaVS}_{3}$ was shown to decrease with increasing local Coulomb interaction. ${ }^{9}$ Also, the Mott transition in $\mathrm{LaVO}_{3}$ and
$\mathrm{YVO}_{3}$ occurs before orbital polarization is complete. ${ }^{10}$ Moreover, in a hypothetical tetragonal structure of $\mathrm{LaTiO}_{3}$, relevant for heterostructures, the Mott phase is reached when $n_{x z, y z}$ approaches $1 / 4$ and $n_{x y}$ vanishes. ${ }^{11}$ Finally, the possibility of so-called orbital-selective Mott transitions in multiband systems has been discussed extensively in the literature. ${ }^{7,12-16}$ These different trends underline the remarkably rich physics of Mott transitions in multiorbital materials.

The aim of this work is to investigate the relationship between Coulomb correlations in single-site multiorbital systems as described above to those occurring within a single band when intersite Coulomb correlations are taken into account. The influence of short-range correlations on the nature of the Mott transition is currently of great interest and has been studied by many groups. ${ }^{17-51}$ Here we examine the role of correlation-driven orbital polarization in the vicinity of the Mott transition. For example, it is well known that in a minimal two-site cluster model, ${ }^{47}$ which permits explicit treatment of short-range Coulomb correlations in an isotropic square lattice, the Green's function and self-energy become diagonal if one transforms the site basis to a diagonal bonding-antibonding molecular orbital basis. In a four-site cluster model, diagonality is obtained by transforming sites to cluster molecular orbitals characterized by $\Gamma=(0,0), X$ $=(\pi, 0),(0, \pi)$, and $M=(\pi, \pi) .{ }^{44}$ The molecular-orbital components of the self-energy provide qualitative information on the importance of correlations in the corresponding sections of the Brillouin Zone. In the case of an isotropic triangular lattice, Green's function and self-energy can be diagonalized by an analogous transformation to molecular orbitals appropriate for a three-site cluster. ${ }^{45}$ The question then arises 
whether these cluster molecular orbitals in the single-band case obey a similar scenario as the multiorbital systems mentioned above.

Since an approximate momentum variation in the lattice self-energy in these models can be derived from a linear superposition of the respective molecular-orbital components of the cluster self-energy, the effect of correlation-enhanced orbital polarization is of direct relevance for the question of whether the Mott gap opens uniformly across the Fermi surface, or whether it opens first in certain regions of the Brillouin Zone (e.g., near the so-called hot spots) and only at larger $U$ in the remaining regions (the so-called cold spots). The latter picture would be analogous to the orbital-selective Mott transition, which can occur within single-site DMFT treatments of certain multiband systems. ${ }^{7,12-16}$ Another possibility, analogous to multiorbital materials such as $\mathrm{LaTiO}_{3}$, $\mathrm{V}_{2} \mathrm{O}_{3}$, and $\mathrm{Ca}_{2} \mathrm{RuO}_{4}$, is that a subset of cluster orbitals could exhibit a genuine Mott transition, while the remaining ones are pushed above or below the Fermi level at about the same critical $U$.

To account for intersite correlations we use DMFT combined with finite-temperature exact diagonalization (ED) ${ }^{52} \mathrm{It}$ was recently shown ${ }^{53}$ that this method can be generalized to multiband materials by computing only those excited states of the impurity Hamiltonian that are within a narrow range above the ground state, where the Boltzmann factor provides the convergence criterion. Exploiting the sparseness of the Hamiltonian, these states can be computed very efficiently by using the Arnoldi algorithm. ${ }^{54}$ Higher excited states enter via Green's functions, which are evaluated using the Lanczos method. This approach has proved to be highly useful for the study of strong correlations in several transition-metal oxides. ${ }^{4,11,53,55,56}$ An important feature of ED/DMFT is that low temperatures and large Coulomb energies can be reached. The adaptation of single-site multiorbital ED to multisite single-band systems is discussed in detail below. In particular, we introduce a mixed site-molecular-orbital basis, which permits a more flexible and more accurate projection of the lattice Green's function onto the cluster than in a pure site representation. Previous multisite ED/DMFT studies focused on $T=0 .{ }^{29,35,37-41}$ The extension to finite $T$ discussed here is especially useful for the evaluation of the $T / U$ phase diagram.

The main result of this work is that in all cluster models studied here for half-filled square and triangular lattices, there is little enhancement of orbital polarization in the vicinity of the Mott transition. Thus, despite sizable level splitting between these cluster orbitals, they all exhibit Mott gaps at the same critical Coulomb energy. As a consequence, the Mott gap in these models opens uniformly across the Fermi surface. For the square lattice we show explicitly that the Mott gap at the cold spot $M / 2=(\pi / 2, \pi / 2)$ of the Brillouin Zone is driven by Coulomb correlations at the hot spot $X$ $=(\pi, 0)$. Therefore, there is no orbital-selective Mott transition. Moreover, there is no evidence for the combination of partial band filling and Mott transition in remaining subbands that is characteristic of single-site DMFT treatments of the multiorbital materials $\mathrm{LaTiO}_{3}, \mathrm{~V}_{2} \mathrm{O}_{3}$, and $\mathrm{Ca}_{2} \mathrm{RuO}_{4}$, as mentioned above.

The outline of this paper is as follows. Section II discusses the theoretical aspects of our cluster ED/DMFT implementation of finite-temperature exact diagonalization. Section III provides the results for the two-site and four-site clusters of the square lattice, and the three-site cluster of the isotropic triangular lattice. In Sec. IV we briefly discuss analogies and differences between these multisite correlation effects and those investigated previously in single-site DMFT treatments of multiorbital materials. The conclusions are presented in Sec. V.

\section{MULTISITE ED/DMFT}

Let us consider the single-band Hubbard model

$$
H=-t \sum_{\langle i j\rangle \sigma}\left(c_{i \sigma}^{+} c_{j \sigma}+H . c .\right)+U \sum_{i} n_{i \uparrow} n_{i \downarrow},
$$

where the sum in the first term extends over nearest-neighbor sites. The hopping integral $t$ will be set equal to unity throughout this paper. Thus, the band widths of the square and triangular lattices are $W=8$ and $W=9$, respectively. Within cellular dynamical mean-field theory (CDMFT) (Refs. 25 and 36) the interacting lattice Green's function in the cluster site basis is given by

$$
G_{i j}\left(i \omega_{n}\right)=\sum_{\vec{k}}\left[i \omega_{n}+\mu-t(\vec{k})-\Sigma\left(i \omega_{n}\right)\right]_{i j}^{-1},
$$

where the $\vec{k}$ sum extends over the reduced Brillouin Zone, $\omega_{n}=(2 n+1) \pi k_{B} T$ are Matsubara frequencies, and $\mu$ is the chemical potential. The lattice constant is unity. $t(\vec{k})$ denotes the hopping matrix for the superlattice and $\Sigma\left(i \omega_{n}\right)$ represents the cluster self-energy matrix. To make contact to other recent works, ${ }^{32,41,44,46,47}$ we consider here the paramagnetic metal-insulator transition.

In the site basis, the Green's functions for two-site, threesite, and four-site clusters have the structure

$$
\begin{gathered}
G^{(2)}=\left(\begin{array}{ll}
a & b \\
b & a
\end{array}\right), \\
G^{(3)}=\left(\begin{array}{lll}
a & b & b \\
b & a & b \\
b & b & a
\end{array}\right), \\
G^{(4)}=\left(\begin{array}{llll}
a & b & b & c \\
b & a & c & b \\
b & c & a & b \\
c & b & b & a
\end{array}\right)
\end{gathered}
$$

with $a=G_{11}, b=G_{12}$, and $c=G_{14}$. Site labels in the square lattice refer to $1 \equiv(0,0), 2 \equiv(1,0), 3 \equiv(0,1)$, and 4 $\equiv(1,1)$, and in the triangular lattice to $1 \equiv(0,0), 2 \equiv(1,0)$, and $3 \equiv(1 / 2, \sqrt{3} / 2)$. The superscript denotes the cluster size $n_{c}$ in the square lattice $\left(n_{c}=2\right.$ or $\left.n_{c}=4\right)$ or triangular lattice $\left(n_{c}=3\right)$, respectively. In the site bases, the corresponding self-energy matrices $\Sigma^{\left(n_{c}\right)}\left(i \omega_{n}\right)$ have the same symmetry properties as the Green's functions.

A key aspect of DMFT is that, to avoid double counting of Coulomb interactions in the quantum impurity calculation, 


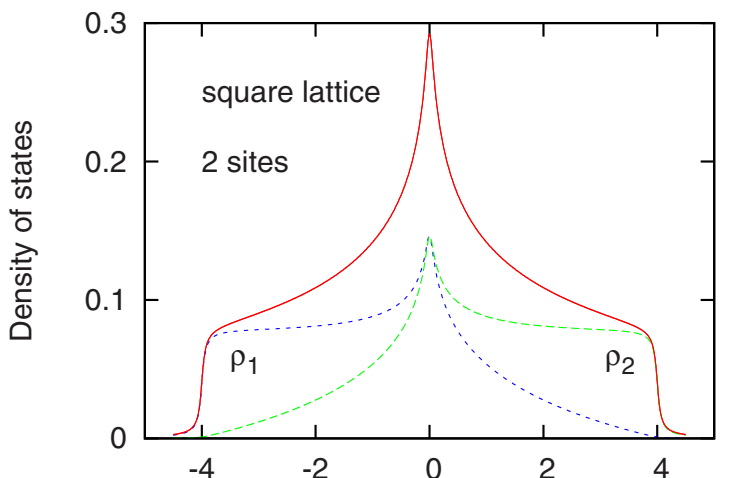

(a)

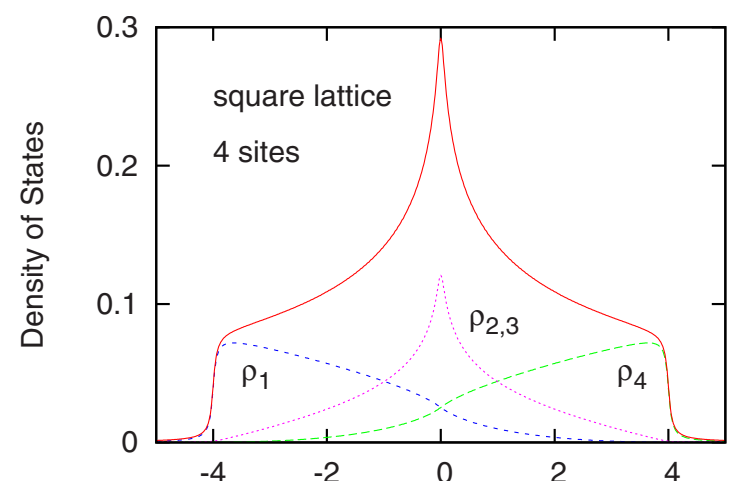

(b)

$\omega$

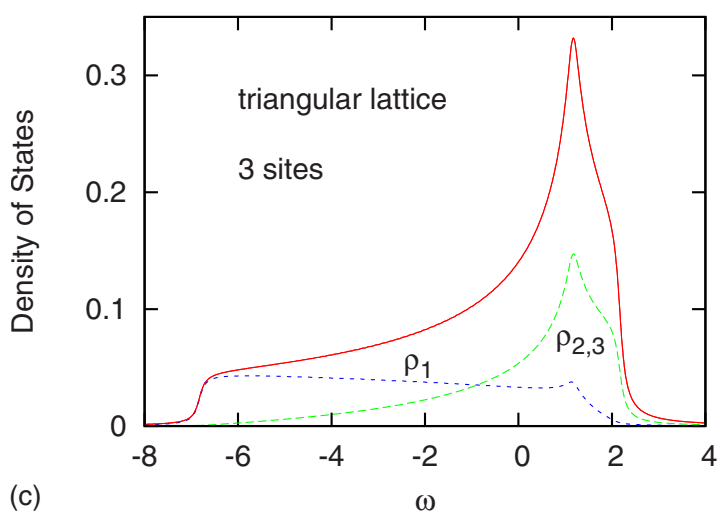

FIG. 1. (Color online) Total density of states $\rho(\omega)$ and molecular-orbital components $\rho_{m}(\omega)$ for two-site and four-site clusters of square lattice (top panels), and of three-site cluster of triangular lattice (bottom panel). For clarity, the molecular-orbital components are divided by $n_{c}$.

it is necessary to remove the self-energy from the cluster in which correlations are treated exactly. This removal yields the Green's function

$$
G_{0}\left(i \omega_{n}\right)=\left[G\left(i \omega_{n}\right)^{-1}+\Sigma\left(i \omega_{n}\right)\right]^{-1} .
$$

These matrices also exhibit the symmetry properties specified above.

For the purpose of the ED calculations it is convenient to transform the site bases into molecular-orbital bases in which the Green's functions and self-energies become diagonal. For the two-site cluster, molecular orbitals are given by the bonding-antibonding combinations $\phi_{1,2}=(|1\rangle \pm|2\rangle) / \sqrt{2}$. For the four-site cluster, they are formed by the plaquettes $\phi_{1}$ $=(|1\rangle+|2\rangle+|3\rangle+|4\rangle) / 2, \quad \phi_{2}=(|1\rangle+|2\rangle-|3\rangle-|4\rangle) / 2, \quad \phi_{3}=(|1\rangle$ $-|2\rangle+|3\rangle-|4\rangle) / 2$, and $\phi_{4}=(|1\rangle-|2\rangle-|3\rangle+|4\rangle) / 2$. Finally, for the three-site cluster of the triangular lattice they can be written as: $\phi_{1}=(|1\rangle+|2\rangle+|3\rangle) / \sqrt{3}, \phi_{2}=(-2|1\rangle+|2\rangle+|3\rangle) / \sqrt{6}$, and $\phi_{3}=(|2\rangle-|3\rangle) / \sqrt{2}$. In these cluster molecular-orbital bases, the above Green's functions take the form

$$
\begin{gathered}
G^{(2)}=\left(\begin{array}{cc}
a+b & 0 \\
0 & a-b
\end{array}\right), \\
G^{(3)}=\left(\begin{array}{ccc}
a+2 b & 0 & 0 \\
0 & a-b & 0 \\
0 & 0 & a-b
\end{array}\right),
\end{gathered}
$$

$$
G^{(4)}=\left(\begin{array}{cccc}
a+2 b+c & 0 & 0 & 0 \\
0 & a-c & 0 & 0 \\
0 & 0 & a-c & b \\
0 & 0 & 0 & a-2 b+c
\end{array}\right) .
$$

The self-energies $\Sigma\left(i \omega_{n}\right)$ and Green's functions $G_{0}\left(i \omega_{n}\right)$ can be diagonalized in the same fashion. We denote these elements as $G_{m}\left(i \omega_{n}\right), \Sigma_{m}\left(i \omega_{n}\right)$, and $G_{0, m}\left(i \omega_{n}\right)$.

In the site basis, the local density of states in the noninteracting limit is given by

$$
\rho_{i i}(\omega)=-\frac{1}{\pi} \operatorname{Im} G_{i i}(\omega)
$$

with $\Sigma=0$. Since we consider isotropic clusters, all sites are equivalent so that $\rho_{i i}(\omega)$ coincides with the density of states $\rho(\omega)$. In the molecular-orbital basis, the density-of-states components $\rho_{m}(\omega)$ have different shapes and different centroids, analogous to the crystal-field-split density-of-states components of many transition-metal oxides. Figure 1 shows these densities for the two-site and four-site clusters of the square lattice and the three-site cluster of the triangular lattice, as described above. According to Eqs. (7)-(9), the cluster molecular-orbital densities of states are given by

$$
\begin{aligned}
& \rho_{1}^{(2)}=\rho_{11}^{(2)}+\rho_{12}^{(2)}, \\
& \rho_{2}^{(2)}=\rho_{11}^{(2)}-\rho_{12}^{(2)},
\end{aligned}
$$




$$
\begin{gathered}
\rho_{1}^{(3)}=\rho_{11}^{(3)}+2 \rho_{12}^{(3)}, \\
\rho_{2}^{(3)}=\rho_{3}^{(3)}=\rho_{11}^{(3)}-\rho_{12}^{(3)}, \\
\rho_{1}^{(4)}=\rho_{11}^{(4)}+2 \rho_{12}^{(4)}+\rho_{14}^{(4)}, \\
\rho_{2}^{(4)}=\rho_{3}^{(4)}=\rho_{11}^{(4)}-\rho_{14}^{(4)}, \\
\rho_{4}^{(4)}=\rho_{11}^{(4)}-2 \rho_{12}^{(4)}+\rho_{14}^{(4)},
\end{gathered}
$$

where $\rho_{i j}^{\left(n_{c}\right)}$ are the site components for cluster $n_{c}$.

From these cluster molecular-orbital densities of states an approximate momentum variation across the Brillouin Zone can be constructed (see below). For instance, in the case of the square lattice with $n_{c}=4$, densities associated with the high-symmetry points of the original lattice are given by $\rho_{\Gamma}(\omega)=\rho_{1}(\omega), \rho_{X}(\omega)=\rho_{2}(\omega)=\rho_{3}(\omega)$, and $\rho_{M}(\omega)=\rho_{4}(\omega)$. At $M / 2=(\pi / 2, \pi / 2)$, the density of states corresponds to the local density $\rho(\omega)=\rho_{11}(\omega)=\left(\rho_{\Gamma}+\rho_{M}+2 \rho_{X}\right) / 4$. Note, however, that all molecular-orbital densities extend across the entire band width. Thus, they are not identical with those sections of the local density of states that originate in momentum regions surrounding the high-symmetry points, as would be the case in the dynamical cluster approximation (DCA). ${ }^{20,36}$

We now project the Green's function $G_{0}\left(i \omega_{n}\right)$ defined in Eq. (6) onto a cluster consisting of $n_{c}$ impurity levels and $n_{b}$ bath levels. The total number of levels is $n_{s}=n_{c}+n_{b}$. In the site basis we have

$$
G_{0}\left(i \omega_{n}\right) \approx G_{0}^{c l}\left(i \omega_{n}\right)=\left[i \omega_{n}+\mu-h-\Gamma\left(i \omega_{n}\right)\right]^{-1}
$$

where $h$ is the noninteracting impurity cluster Hamiltonian and $\Gamma\left(i \omega_{n}\right)$ is the hybridization matrix describing the coupling between impurity cluster and bath. Thus,

$$
\begin{gathered}
h^{(2)}=\left(\begin{array}{ll}
\epsilon_{0} & t \\
t & \epsilon_{0}
\end{array}\right), \\
h^{(3)}=\left(\begin{array}{lll}
\epsilon_{0} & t & t \\
t & \epsilon_{0} & t \\
t & t & \epsilon_{0}
\end{array}\right), \\
h^{(4)}=\left(\begin{array}{llll}
\epsilon_{0} & t & t & 0 \\
t & \epsilon_{0} & 0 & t \\
t & 0 & \epsilon_{0} & t \\
0 & t & t & \epsilon_{0}
\end{array}\right) .
\end{gathered}
$$

For the square lattice we choose $\epsilon_{0}=0$ and for the triangular lattice $\epsilon_{0}=-0.83$ so that the Fermi level coincides with $\omega$ $=0$.

Instead of expressing the nondiagonal hybridization matrix $\Gamma\left(i \omega_{n}\right)$ in a site basis, it is convenient to go over to the molecular-orbital basis in which $G_{0}\left(i \omega_{n}\right)$ is diagonal. Assuming that each component $G_{0, m}\left(i \omega_{n}\right)$ couples only with its own bath, we have

$$
G_{0, m}\left(i \omega_{n}\right) \approx G_{0, m}^{c l}\left(i \omega_{n}\right)=\left(i \omega_{n}+\mu-\epsilon_{m}-\sum_{k} \frac{\left|V_{m k}\right|^{2}}{i \omega_{n}-\epsilon_{k}}\right)^{-1},
$$

where $\epsilon_{m}$ represents an impurity level, $\epsilon_{k}$ represent the bath levels, and $V_{m k}$ represent the hybridization matrix elements. The incorporation of the impurity level $\epsilon_{m}$ ensures a much better fit of $G_{0, m}\left(i \omega_{n}\right)$ than by projecting only onto bath orbitals.

For instance, for $n_{c}=3$ and $n_{s}=12$ (i.e., three bath levels per impurity orbital), each component $G_{0, m}\left(i \omega_{n}\right)$ is fitted using seven parameters: one impurity level $\epsilon_{m}$, three bath levels $\epsilon_{k}$, and three hopping integrals $V_{m k}$. Since according to Eq. (8) there are two independent functions, we use a total of 14 fit parameters to represent these two $G_{0, m}$ components. This procedure allows for a considerably more flexible projection of the Green's function matrix $G_{0}\left(i \omega_{n}\right)$ onto the bath. In a site basis for an isotropic triangular lattice (taking again three bath levels per site), one would have instead only six fit parameters if each site couples to its own bath. Effectively, therefore, the molecular-orbital basis accounts for several additional cross hybridization terms, as well as internal cluster couplings (see below). Moreover, it is much more reliable to fit the two independent molecular-orbital components $G_{0, m}\left(i \omega_{n}\right)$ than a nondiagonal site matrix $G_{0, i j}\left(i \omega_{n}\right)$ with an equivalent number of parameters. Analogous considerations hold for the two-site and four-site clusters of the square lattice. For example, for $n_{c}=4$ and $n_{s}=12$ there are two independent functions $G_{0,1}$ and $G_{0,2}\left(G_{0,4}\right.$ is related to $\left.G_{0,1}\right)$, giving a total of ten fit parameters, compared to only two parameters in a simple site picture with fourforld and particle-hole symmetry.

Figure 2 illustrates the typical quality of the projection of the lattice components of $G_{0, m}\left(i \omega_{n}\right)$ onto the bath for $n_{c}=3$ and $n_{s}=9$. Thus, although only two bath levels per orbital are included (i.e., using five parameters per orbital), the fit of both real and imaginary parts is excellent. For these cluster sizes and low temperatures, iterations take only a few minutes. Fits of similar quality are achieved for multiorbital materials. $^{4,11,53,55,56}$ To achieve even better agreement at low frequencies, it is preferable to minimize not the bare difference $G_{0, m}\left(i \omega_{n}\right)-G_{0, m}^{c l}\left(i \omega_{n}\right)$ but to divide these functions first by $\omega_{n}$.

We now discuss the evaluation of the finite-temperature interacting cluster Green's function. If this step is carried out in the diagonal molecular-orbital basis, the Coulomb interaction must be expressed as a matrix containing many interorbital components. For $n_{c}=4$ it can be easily shown that $U_{m_{1} m_{2} m_{3} m_{4}}=U / 4$ for 64 of the possible 256 configurations. All other matrix elements vanish. This step can be circumvented by working in a mixed basis consisting of cluster sites $i$ and bath orbitals $k$. We illustrate this procedure here for the triangular lattice with $n_{c}=3$. Let us denote the transformation between sites and orbitals as $T^{\left(n_{c}\right)}$, where

$$
T_{i m}^{(3)}=\left(\begin{array}{ccc}
1 / \sqrt{3} & -2 / \sqrt{6} & 0 \\
1 / \sqrt{3} & 1 / \sqrt{6} & 1 / \sqrt{2} \\
1 / \sqrt{3} & 1 / \sqrt{6} & -1 / \sqrt{2}
\end{array}\right) .
$$

In this mixed basis, the effective site block of the cluster Hamiltonian becomes 

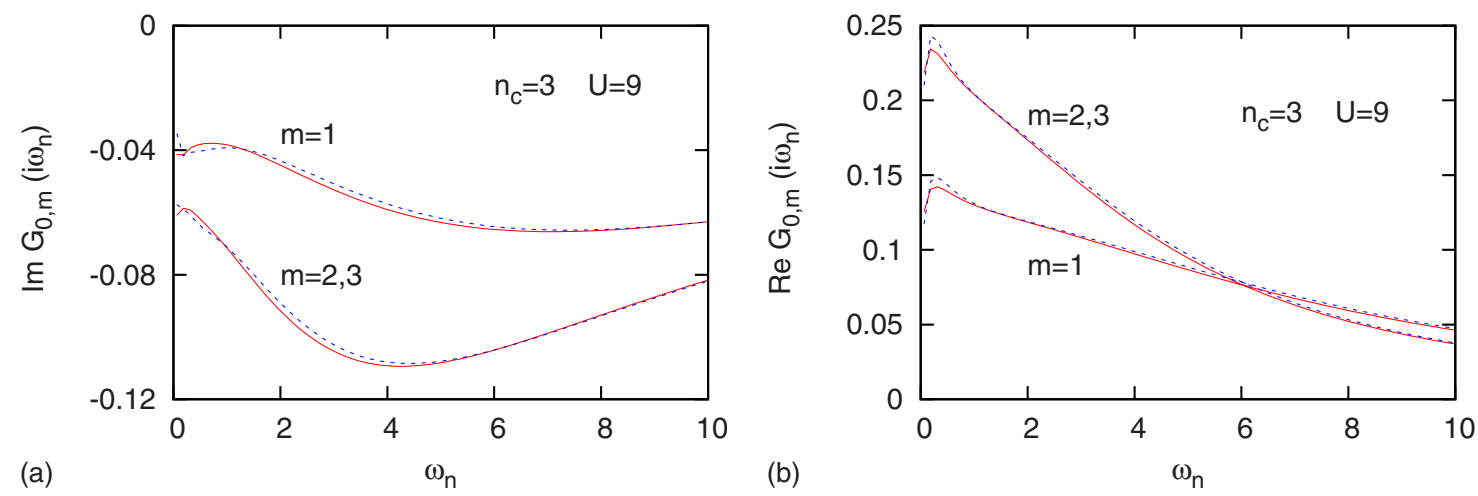

FIG. 2. (Color online) Projection of lattice Green's function components $G_{0, m}\left(i \omega_{n}\right)$ onto bath for $n_{c}=3, U=9, T=0.02$. Left panel: $\operatorname{Im} G_{0, m}$, right panel: $\operatorname{Re} G_{0, m}$. Red curves: lattice Green's functions, blue curves: approximate expression, right-hand side of Eq. (18).

$$
h^{(3)}=\left(\begin{array}{ccc}
\epsilon^{\prime} & t^{\prime} & t^{\prime} \\
t^{\prime} & \epsilon^{\prime} & t^{\prime} \\
t^{\prime} & t^{\prime} & \epsilon^{\prime}
\end{array}\right)
$$

with $\epsilon^{\prime}=\epsilon_{0}+\left(\epsilon_{1}+2 \epsilon_{2}\right) / 3$ and $t^{\prime}=t+\left(\epsilon_{1}-\epsilon_{2}\right) / 3$, where $\epsilon_{0}$ and $t$ are the elements of the original cluster Hamiltonian defined in Eq. (16). The new terms involving the molecular-orbital cluster levels $\epsilon_{m}$ arise from the projection specified in Eq. (18). In the mixed basis, the hybridization matrix elements $V_{m k}$ between cluster and bath orbitals introduced in Eq. (18) are transformed to new hybridization matrix elements between cluster sites $i$ and bath orbitals $k$. They are given by

$$
V_{i k}^{\prime}=\left(T^{(3)} V\right)_{i k}=\sum_{m} T_{i m}^{(3)} V_{m k}
$$

Using the elements $\epsilon^{\prime}, t^{\prime}$, and $V_{i k}^{\prime}$, together with the onsite Coulomb energy $U$, the nondiagonal interacting cluster Green's function at finite temperature is derived from the expression $^{53,57}$

$$
\begin{aligned}
G_{i j}^{c l}\left(i \omega_{n}\right)= & \frac{1}{Z} \sum_{\nu \mu} e^{-\beta E_{\nu}}\left(\frac{\left\langle\nu\left|c_{i \sigma}\right| \mu\right\rangle\left\langle\mu\left|c_{j \sigma}^{+}\right| \nu\right\rangle}{E_{\nu}-E_{\mu}+i \omega_{n}}\right. \\
& \left.+\frac{\left\langle\nu\left|c_{i \sigma}^{+}\right| \mu\right\rangle\left\langle\mu\left|c_{j \sigma}\right| \nu\right\rangle}{E_{\mu}-E_{\nu}+i \omega_{n}}\right),
\end{aligned}
$$

where $E_{\nu}$ and $|\nu\rangle$ denote the eigenvalues and eigenvectors of the impurity Hamiltonian, and $\beta=1 / k_{B} T$ and $Z$ $=\Sigma_{\nu} \exp \left(-\beta E_{\nu}\right)$ is the partition function. At low temperatures only a relatively small number of excited states in few spin sectors contributes to $G_{i j}^{c l}$. They can be efficiently evaluated using the Arnoldi algorithm. ${ }^{54}$ The excited state Green's functions are computed using the Lanczos procedure. Further details can be found in Ref. 53. The nondiagonal elements of $G_{i j}^{c l}$ are derived by first evaluating the diagonal components $G_{i i}^{c l}$ and then using the relation ${ }^{50}$

$$
G_{(i+j)(i+j)}^{c l}=G_{i i}^{c l}+G_{i j}^{c l}+G_{j i}^{c l}+G_{j j}^{c l} .
$$

Since $G_{i j}^{c l}=G_{j i}^{c l}$, this yields

$$
G_{i j}^{c l}=\frac{1}{2}\left(G_{(i+j)(i+j)}^{c l}-G_{i i}^{c l}-G_{j j}^{c l}\right) .
$$

For the two-site cluster, we have used three or four bath levels for each impurity orbital $\left(n_{s}=8\right.$ or 10$)$, for the threesite cluster 2 or 3 bath levels per impurity orbital $n_{s}=9$ or 12), and for the four-site cluster 2 bath levels per impurity orbital $\left(n_{s}=12\right)$. $G_{i j}^{c l}\left(i \omega_{n}\right)$ obeys the same symmetry properties as the lattice Green's functions given in Eqs. (3)-(5). It can therefore be diagonalized as indicated in Eqs. (7)-(9). We denote these diagonal elements as $G_{m}^{c l}\left(i \omega_{n}\right)$. For $n_{c}=4$, we have checked that the evaluations of $G_{m}^{c l}\left(i \omega_{n}\right)$ in the nondiagonal site-orbital basis and in the diagonal molecularorbital basis yield identical results.

The key assumption in DMFT is now that the resulting impurity cluster self-energy is a physically reasonable representation of the lattice self-energy. Thus, using a relation analogous to Eq. (6), we find

$$
\Sigma_{m}^{c l}\left(i \omega_{n}\right)=1 / G_{0, m}^{c l}\left(i \omega_{n}\right)-1 / G_{m}^{c l}\left(i \omega_{n}\right) \approx \Sigma_{m}\left(i \omega_{n}\right) .
$$

After transforming $\Sigma_{m}\left(i \omega_{n}\right)$ back to the nondiagonal site basis, it is used as input in the lattice Green's function Eq. (2) in the next iteration step.

To summarize the procedure discussed above, the multisite ED/DMFT calculation consists of the following steps:

(a) evaluate the lattice Green's function $G_{i j}\left(i \omega_{n}\right)$, Eq. (2), in the nondiagonal site basis using as input the self-energy obtained in a previous iteration step. The entire iteration procedure is started at small $U$ with $\Sigma=0$.

(b) transform $G_{i j}$ and $\Sigma_{i j}$ to the diagonal molecular-orbital basis and compute the components $G_{0, m}$.

(c) project the $G_{0, m}\left(i \omega_{n}\right)$ onto independent baths to determine $\epsilon_{m}, \epsilon_{k}$, and $V_{m k}$ as indicated in Eq. (18).

(d) from the fit parameters $\epsilon_{m}$ and $V_{m k}$ determine the Hamiltonian matrix elements $\epsilon^{\prime}, t^{\prime}$, and $V_{i k}^{\prime}$ in the mixed site-orbital basis.

(e) evaluate the nondiagonal cluster Green's function $G_{i j}^{c l}\left(i \omega_{n}\right)$ using the Arnoldi and Lanczos methods.

(f) transform this Green's function to the diagonal orbital basis and compute the cluster self-energy components $\Sigma_{m}^{c l}\left(i \omega_{n}\right)$ defined in Eq. (25).

We emphasize that ED/DMFT involves, at each iteration, two projections: (1) The lattice Green's function $G_{0}$ is projected onto the cluster Green's function $G_{0}^{c l}$, as indicated in Eq. (18). By definition, $G_{0}$ has a continuous spectrum at real frequencies, while $G_{0}^{c l}$ is discrete. (2) The cluster self-energy 

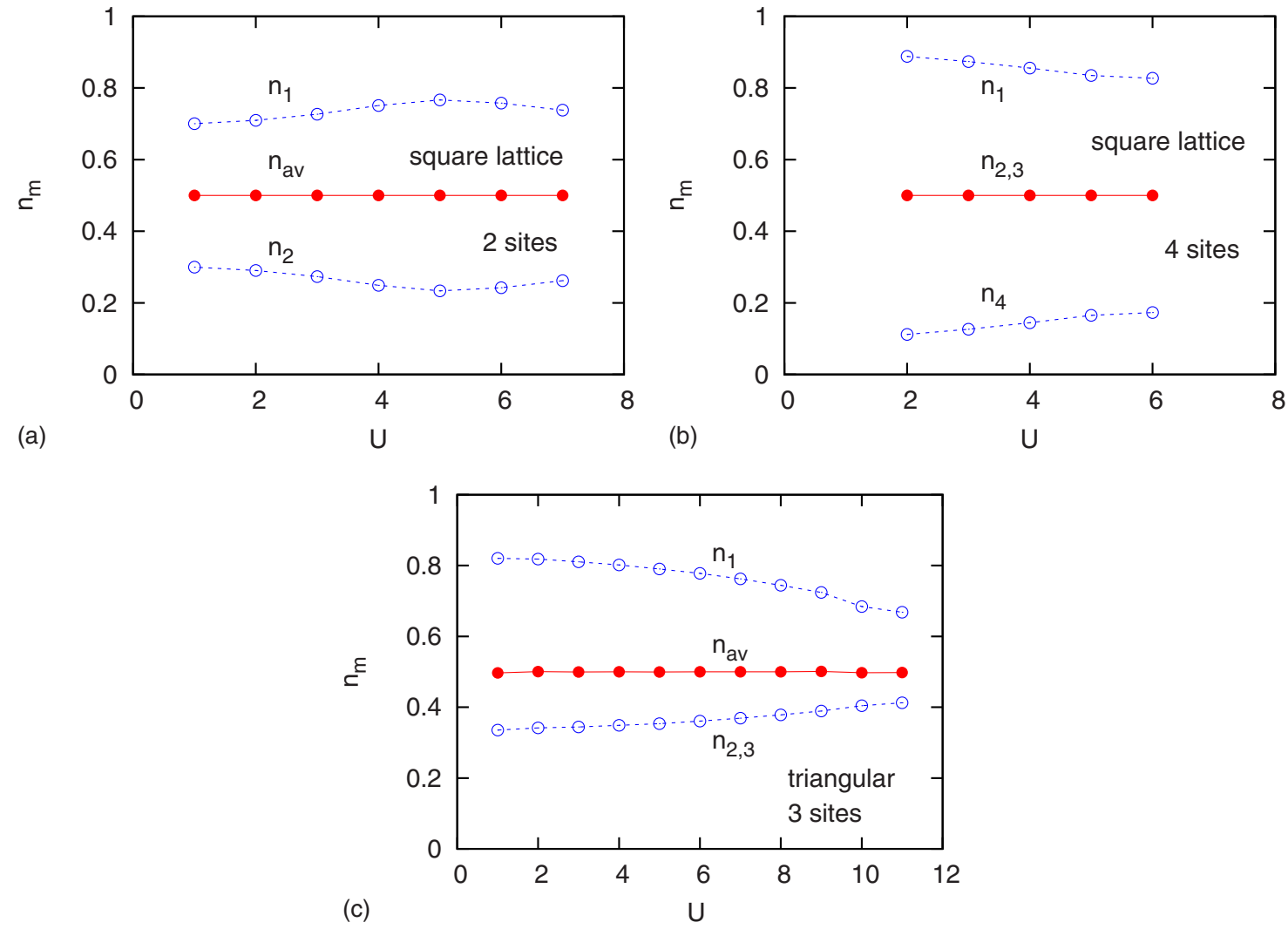

FIG. 3. (Color online) Occupancies of cluster molecular orbitals (per spin) for two- and four-site clusters of square lattice (top panels), and of three-site cluster of triangular lattice (bottom panel) as functions of Coulomb energy $U$. The temperature is $T=0.02$. The sum of these molecular occupancies is $n_{c} / 2$ and their average is 0.5 . The Mott transition for increasing $U$ occurs at $U_{c 2} \approx 5.5$ for the square lattice $\left(n_{c}\right.$ $=2$ and $\left.n_{c}=4\right)$, and near $U_{c 2} \approx 9.5$ for the triangular lattice $\left(n_{c}=3\right)$.

$\Sigma^{c l}$, which evidently has a discrete spectrum at real $\omega$, is used as an approximation of the lattice self-energy $\Sigma$, which by definition is continuous along the real frequency axis. Thus, both projections, $G_{0} \approx G_{0}^{c l}$ and $\Sigma^{c l} \approx \Sigma$, rely on the wellknown fact that continuous and discrete spectra at real $\omega$ can yield nearly identical distributions at Matsubara frequencies. Since the cluster size determines the number of discrete spectral features of $G_{0}^{c l}$ and $\Sigma^{c l}$, there exists evidently an infinite number of discrete spectra, which may in principle be used to represent the continuous spectra of the lattice quantities $G_{0}$ and $\Sigma$.

\section{RESULTS AND DISCUSSION}

Figure 3 shows the occupancies of the cluster molecular orbitals for three cluster sizes as functions of increasing $U$. For the square lattice the Mott transition occurs near $U_{c 2}$ $\approx 5.5$, while for the triangular lattice it occurs near $U_{c 2}$ $\approx 9.5$ (see below). Evidently all orbital occupancies vary smoothly across the transition. There is no indication of orbital-selective Mott transitions or for complete filling or emptying of any orbitals at large Coulomb energies. This behavior differs qualitatively from the one found in materials such as $\mathrm{LaTiO}_{3}, \mathrm{~V}_{2} \mathrm{O}_{3}$, and $\mathrm{Ca}_{2} \mathrm{RuO}_{4}$, where the insulating phase exhibits nearly complete orbital polarization as a result of a Coulomb driven enhancement of the crystal-field splitting between $t_{2 g}$ orbitals ${ }^{3-8}$ (see following section).
The fact that all cluster orbitals remain partially occupied across the transition implies that the gap opens simultaneously in all orbitals. This can be seen most clearly in the spectral distributions, as shown in Fig. 4 for the square lattice with $n_{c}=4$. Since we are here concerned with the transition from metallic to insulating behavior, we show the spectra obtained from the interacting cluster Green's function, $A_{i j}(\omega)=-(1 / \pi) \operatorname{Im} G_{i j}^{c l}(\omega+i \delta)$, Eq. (22), with $\delta=0.1$. These spectra can be evaluated without requiring analytic continuation from Matsubara to real frequencies. Using the transformations indicated in Eqs. (9) and (13), the cluster molecularorbital densities are given by $A_{1}=A_{11}+2 A_{12}+A_{14}, A_{2}=A_{3}$ $=A_{11}-A_{14}$, and $A_{4}=A_{11}-2 A_{12}+A_{14}$. The total density $A(\omega)$ $=\Sigma_{m} A_{m}(\omega) / 4$ coincides with the on-site distribution $A_{11}(\omega)$, which also represents the density corresponding to $M / 2$ $=(\pi / 2, \pi / 2)$ (see below). To our knowledge, this orbital decomposition has not been addressed before. Clearly, all orbitals contribute to the spectral weight at $E_{F}$ in the metallic phase, as well as to the lower and upper Hubbard bands in the insulating phase. The spectral distributions shown in the upper panel are consistent with those by Kyung et al. ${ }^{37}$ and Zhang and Imada ${ }^{41}$ within ED/DMFT at $T=0$. The spectra reveal a characteristic four-peak structure, consisting of lowfrequency peaks limiting the pseudogap due to short-range correlations and high-frequency peaks associated with the Hubbard bands. ${ }^{18,19,37}$ The small peak at $E_{F}$ in the metallic phase at $U=5$ appears only at finite $T$. It vanishes at $T=0$. At 


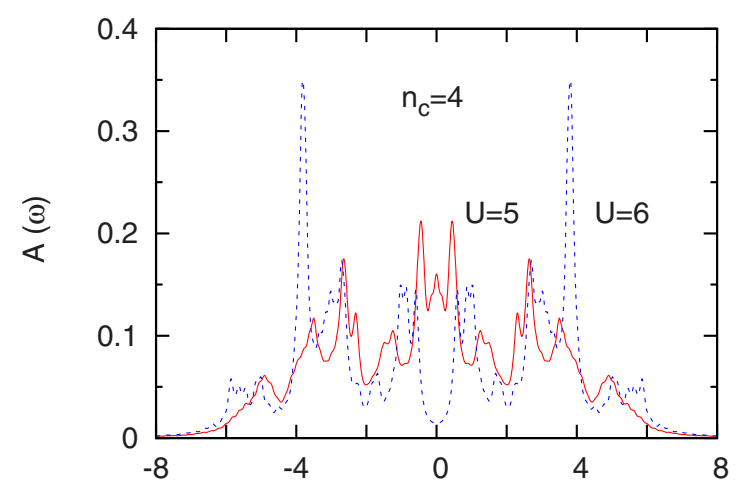

(a)

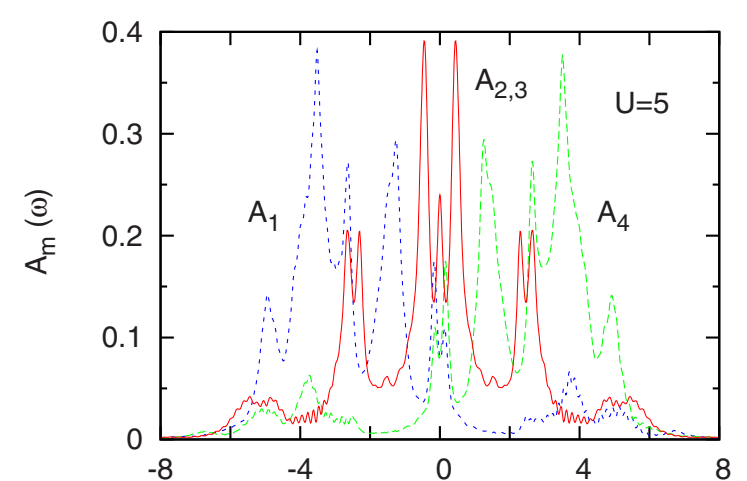

(b)

$\omega$

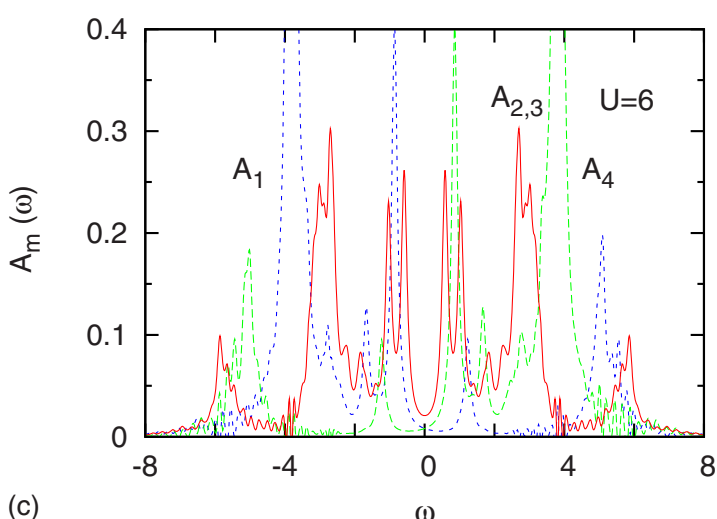

FIG. 4. (Color online) (a) total spectral distribution below and above the Mott transition $\left(U_{c} \approx 5.5\right)$ for the square lattice with $n_{c}=4$ at temperature $T=0.02$ : $U=5$ (red curve) and $U=6$ (blue curve). (b) and (c) molecular-orbital contributions at $U=5$ and $U=6$; blue curves: $A_{1}(\omega)$ (associated with $\Gamma$ ), green curves: $A_{4}(\omega)(M)$, red curves: $A_{2,3}(\omega)(X)$ (broadening $\delta=0.1$ ).

such low frequencies, however, ED finite-size effects cannot be ruled out.

Similar results are obtained for the square lattice in the two-site cluster model. Results for the triangular lattice with $n_{c}=3$ are shown in Fig. 5. According to Eqs. (8) and (12) the cluster molecular-orbital densities are given by $A_{1}=A_{11}$ $+2 A_{12}$ and $A_{2}=A_{3}=A_{11}-A_{12}$. As for the square lattice, the Mott gap opens simultaneously in all orbitals at about the same critical $U$, and all orbitals contribute to the lower and upper Hubbard bands. While the metallic phase of the unfrustrated square lattice close to the transition exhibits a

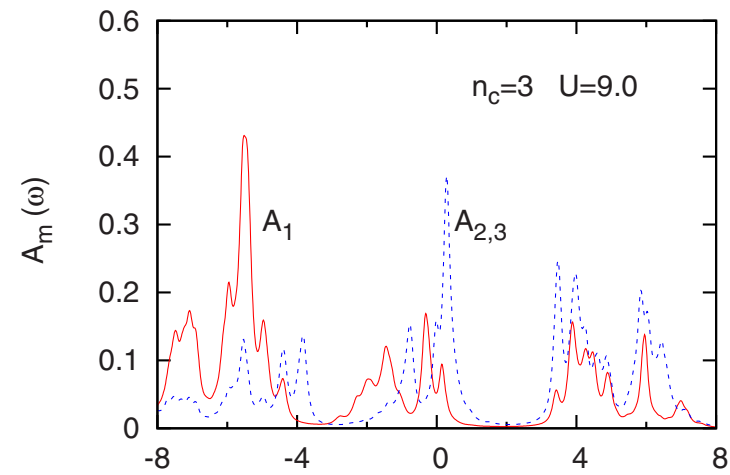

(a)

$\omega$ pseudogap due to short-range antiferromagnetic correlations, ${ }^{37,41,44,46}$ this phenomenon is absent in the triangular lattice as a result of geometrical frustration. ${ }^{39}$

To illustrate the first-order nature of the metal-insulator transition we show in the left panel of Fig. 6 the spectral weight of the $n_{c}=3$ cluster orbitals at $E_{F}=0$ as a function of $U$. The right panel shows the average double occupancy $d_{\text {occ }}=\sum_{m}\left\langle n_{m \uparrow} n_{m \downarrow}\right\rangle / 3$. Both quantities exhibit hysteresis for increasing and decreasing $U$, indicating the coexistence of metallic and insulating solutions. The complete $T / U$ phase diagram will be published elsewhere. The phase diagram for the

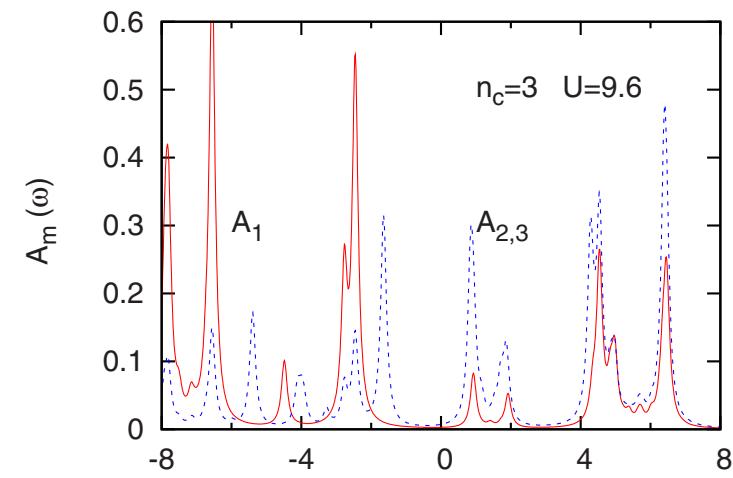

(b)

$\omega$

FIG. 5. (Color online) Spectral distributions of cluster molecular orbitals below and above the Mott transition $\left(U_{c} \approx 9.5\right)$ for the triangular lattice with $n_{c}=3$ at temperature $T=0.02$. Left panel: $U=9.0$; right panel: $U=9.6$. Blue curves: $A_{1}(\omega)$, red curves: $A_{2,3}(\omega)$. 

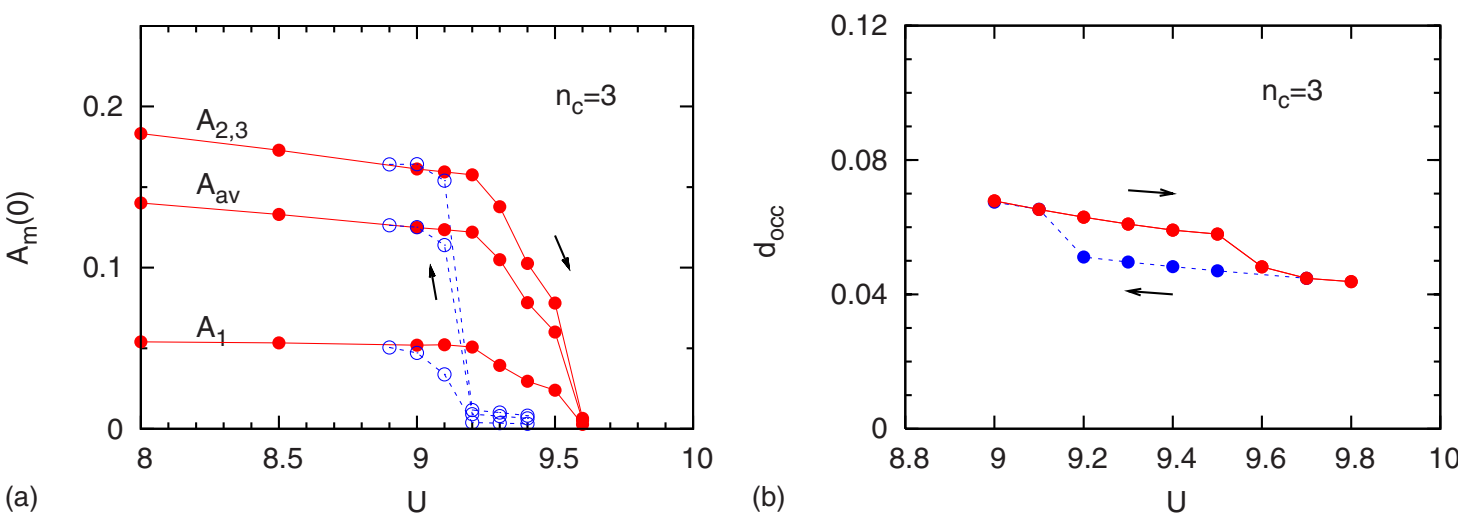

FIG. 6. (Color online) Hysteresis behavior of spectral weights $A_{m}(0)$ of cluster molecular orbitals at $E_{F}=0$ (left panel) and average double occupancy (right panel) as functions of Coulomb energy for triangular lattice with $n_{c}=3(T=0.02)$. Red curves: increasing $U$, blue curves: decreasing $U$.

isotropic square lattice with $n_{c}=4$ was recently mapped out in detail by Park et al. ${ }^{44}$

The cluster molecular-orbital components of the selfenergy and Green's function may be used to derive an approximate expression for the momentum variation of the lattice self-energy and Green's function in the original Brillouin Zone: ${ }^{32}$

$$
\Sigma\left(\vec{k}, i \omega_{n}\right) \approx \frac{1}{n_{c}} \sum_{i j} e^{i \vec{k} \cdot\left(\vec{R}_{i}-\vec{R}_{j}\right)} \Sigma_{i j}\left(i \omega_{n}\right),
$$

where $\vec{R}_{i}$ are the cluster site positions and $\Sigma_{i j}$ are the site components of the self-energy. An analogous expression holds for the lattice Green's function. For the clusters discussed above this superposition implies that the Mott gap opens uniformly along the Fermi surface since all orbitals undergo a common transition.

Writing the site elements in terms of the orbital components, one has for $n_{c}=4: \Sigma\left(\Gamma, i \omega_{n}\right)=\Sigma_{1}\left(i \omega_{n}\right), \quad \Sigma\left(X, i \omega_{n}\right)$ $=\Sigma_{2}\left(i \omega_{n}\right)=\Sigma_{3}\left(i \omega_{n}\right)$, and $\Sigma\left(M, i \omega_{n}\right)=\Sigma_{4}\left(i \omega_{n}\right)$. In agreement with results of previous authors $32,35,41,44,46,47,49$ we find the behavior of $\Sigma\left(\Gamma, i \omega_{n}\right)$ and $\Sigma\left(M, i \omega_{n}\right)$ near the Mott transition to differ qualitatively from that of $\Sigma\left(X, i \omega_{n}\right)$ (not shown here): Whereas $\operatorname{Im} \Sigma\left(X, i \omega_{n}\right)$ exhibits $\sim \omega_{n}$ variation at low frequencies in the metallic phase and $\sim 1 / \omega_{n}$ variation in the insulating phase [the real part vanishes because of particle hole symmetry (see Fig. 1$)], \operatorname{Im} \Sigma\left(\Gamma, i \omega_{n}\right)$ and $\operatorname{Im} \Sigma\left(M, i \omega_{n}\right)$ remain $\sim \omega_{n}$ in both phases, but their real parts increase rapidly across the transition.

Although this behavior might suggest a Mott transition for the $X$ cluster orbitals combined with a band-filling or bandemptying mechanism for the $\Gamma$ and $M$ orbitals, the orbital occupancies (Fig. 3) and spectral distributions (Fig. 4) demonstrate this not to be the case. Moreover, for the crucial question of whether or not the Mott gap opens simultaneously across the Fermi surface, it is important to compare the self-energy at $X$ with its behavior at $M / 2$, where the electron band also crosses $E_{F}$. According to Eq. (26), $\Sigma\left(M / 2, i \omega_{n}\right)$ coincides with the diagonal on-site element of the cluster self-energy, which is identical with the local lattice self-energy. Thus,

$$
\Sigma\left(M / 2, i \omega_{n}\right)=\frac{1}{4}\left[\Sigma\left(\Gamma, i \omega_{n}\right)+\Sigma\left(M, i \omega_{n}\right)+2 \Sigma\left(X, i \omega_{n}\right)\right] .
$$

The real parts of the first terms on the right-hand side cancel since the corresponding density-of-states components are mirrors of each other (see Fig. 1). Thus, $\Sigma\left(M / 2, i \omega_{n}\right)$ and $\Sigma\left(X, i \omega_{n}\right)$ are purely imaginary because of particle-hole symmetry. The above relation demonstrates that Coulomb correlations at the cold spot $M / 2$ are essentially driven by those at the hot spot $X$. In fact, the magnitude of the self-energy at $M / 2$ is a factor of 2 smaller than the singular term at $X$ with weak additional, nonsingular contributions associated with $\Gamma$ and $M$.

Along the Fermi surface between $X$ and $M / 2$ (i.e., for $k_{x}+k_{y}=\pi$ ), the self-energy is given by

$$
\Sigma\left(\vec{k}, i \omega_{n}\right)=\cos ^{2}\left(k_{x}\right) \Sigma\left(X, i \omega_{n}\right)+\sin ^{2}\left(k_{x}\right) \Sigma\left(M / 2, i \omega_{n}\right) .
$$

This function is imaginary, i.e., there are no band shifts due to a finite real part of the self-energy. Thus, within the foursite cluster DMFT, the opening of the Mott gap on the entire Fermi surface is determined solely by the singularity of $\operatorname{Im} \Sigma\left(X, i \omega_{n}\right)$.

The above analysis leads to a surprisingly simple picture for the momentum variation in correlations along the Fermi surface. It consists of two sinusoidal contributions: The singular $X$ term oscillates with amplitude 1 at $X$ and $1 / 2$ at $M / 2$, and the nonsingular term due to the $\Gamma, M$ orbitals oscillates with amplitude 1 at $M / 2$ and zero at $X$.

This is illustrated in Fig. 7, which shows the lattice selfenergy and Green's function at $X$ and $M / 2$ below and above the Mott transition. In the metallic phase, the self-energy at $X$ is seen to be larger than the one at $M / 2$. Thus, quasiparticle lifetimes decrease between $M / 2$ and $X$. Nevertheless, the singular behavior at $M / 2$ is governed by the one at $X$. Accordingly, the Green's function at $X$ and $M / 2$ displays a change from metallic to insulating behavior at the same Coulomb energy. 

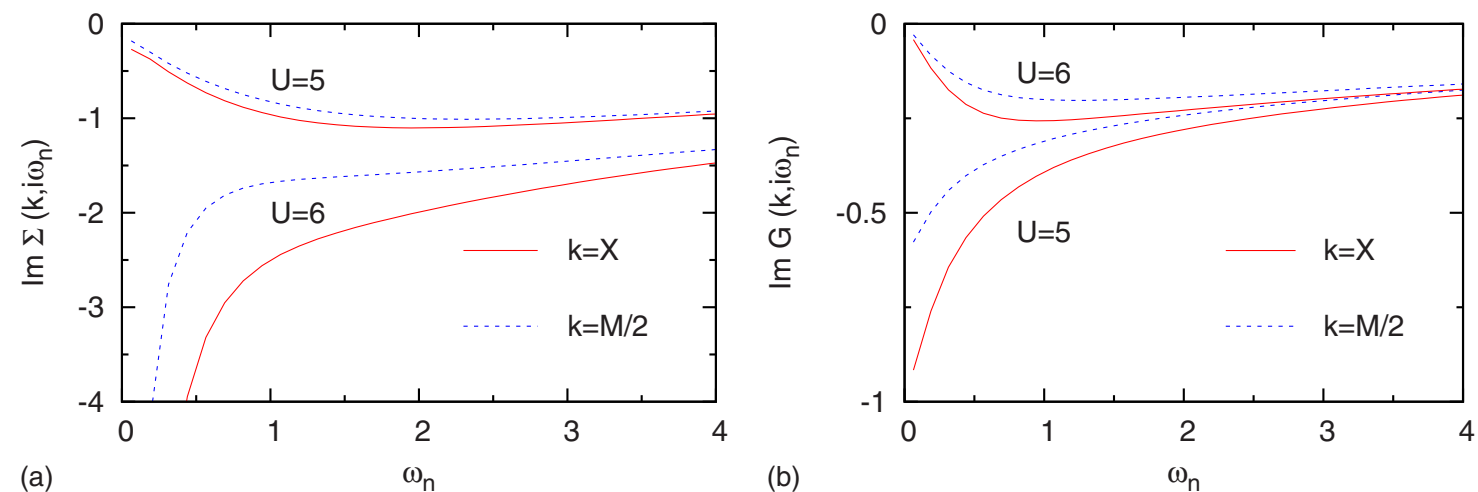

FIG. 7. (Color online) Lattice self-energy, Eq. (26), (left panel) and Green's function (right panel) at $X=(\pi, 0)($ red curves) and $M / 2$ $=(\pi / 2, \pi / 2)$ (blue curves) for metallic phase $(U=5)$ and insulation phase $(U=6)$ of square lattice $\left(n_{c}=4\right)$ at $T=0.02$. Both $\Sigma\left(\vec{k}, i \omega_{n}\right)$ and $G\left(\vec{k}, i \omega_{n}\right)$ are purely imaginary between $X$ and $M / 2$. The sinusoidal variation in the self-energy between these points is given by Eq. (28).

To understand the strong coupling between different sections of the Brillouin Zone it is important to recall that, in the diagonal cluster molecular-orbital basis, the single-particle part of the lattice Hamiltonian appearing in Eq. (2) is not diagonal. Thus, the orbital component of the lattice Green's function $G_{m}\left(i \omega_{n}\right)$ is influenced not only by the corresponding self-energy $\Sigma_{m}\left(i \omega_{n}\right)$, but by the other orbital elements as well. This point becomes clear if we compare $G_{m}\left(i \omega_{n}\right)$ with the approximation

$$
G_{m}\left(i \omega_{n}\right) \approx \int d \omega \frac{\rho_{m}(\omega)}{i \omega_{n}+\mu-\omega-\Sigma_{m}\left(i \omega_{n}\right)} .
$$

Figure 8 shows that in the metallic region at $U=5$ there is little difference with regard to the actual $G_{m}\left(i \omega_{n}\right)$. Also, at $U=6$ the key components responsible for the metal-insulator transition, namely, $G_{2,3}\left(i \omega_{n}\right)$ corresponding to $X=(\pi, 0)$, are well represented by this approximation. The $\Gamma, M$ components, $G_{1,4}\left(i \omega_{n}\right)$, however, do not reveal insulating behavior since at small $\omega_{n}$ the imaginary parts do not extrapolate to zero. This demonstrates that the Mott gaps seen in $A_{1,4}(\omega)$ in Figs. 4 and 5 are not caused by the rapidly varying real parts of $\Sigma_{1,4}\left(i \omega_{n}\right)$. Instead, the gaps at $\Gamma$ and $M$ are driven by the singular behavior of $\operatorname{Im} \Sigma_{2,3}\left(i \omega_{n}\right)$, which contributes to $A_{1,4}(\omega)$ via the nondiagonal elements of $t(\vec{k}) .{ }^{58}$

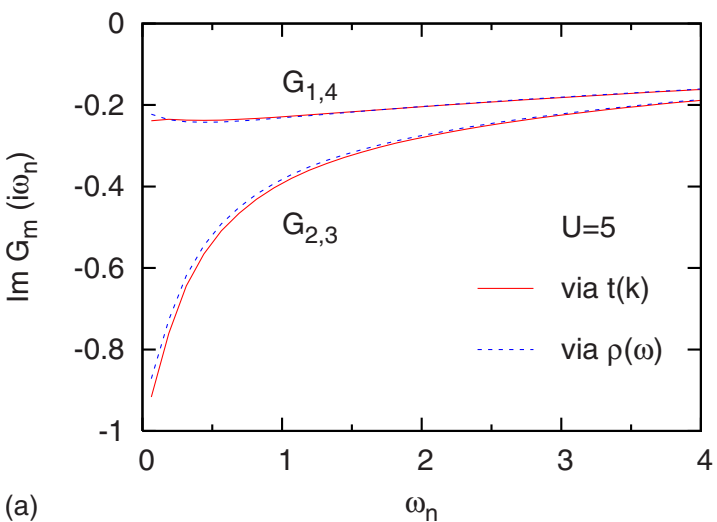

If the approximate components obtained via Eq. (29) were used to generate the spectral distributions $A_{m}(\omega)$, it is clear that only $A_{2,3}(\omega)$ corresponding to $X$ would exhibit the Mott transition, while $A_{1,4}(\omega)$ would retain considerable metallicity. This implies that the physics at the cold spot $M / 2$ is incorrectly represented via Eq. (29), suggesting that the gap at $M / 2$ does not open at the same $U$ as at $X$. Instead, as argued above, the metal-insulator transition at $M / 2$ is caused by the same self-energy terms as at $X$, i.e., the Mott gap opens uniformly.

As pointed out earlier, in the molecular-orbital basis the Coulomb matrix has a large number of nonzero elements. Thus, in this basis there is not only single-particle hybridization arising from $t(\vec{k})$, but also strong interorbital Coulomb repulsion. Nevertheless, the above analysis reveals that, although these Coulomb interaction terms are properly taken into account, the spectral distributions at $\Gamma, M$, and $M / 2$ do not exhibit a Mott gap unless the nondiagonal terms of $t(\vec{k})$ in the orbital basis are included. It is therefore the singleparticle part of the Hamiltonian that provides the correct connection between the self-energy components and thereby generates the true momentum variation in the spectral distribution of the single band.

Because of the strong coupling between orbitals, the notion that some of these orbitals undergo a Mott transition

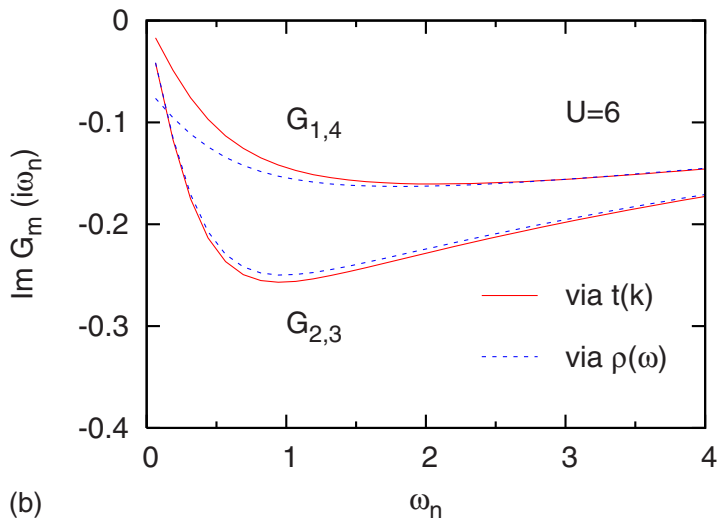

FIG. 8. (Color online) Lattice Green's function Eq. (2) in $n_{c}=4$ cluster molecular-orbital basis for $U=5$ (left panel) and $U=6$ (right panel) at $T=0.02$. Red curves: diagonal version of Eq. (2); blue curves: approximate expression, Eq. (29). 


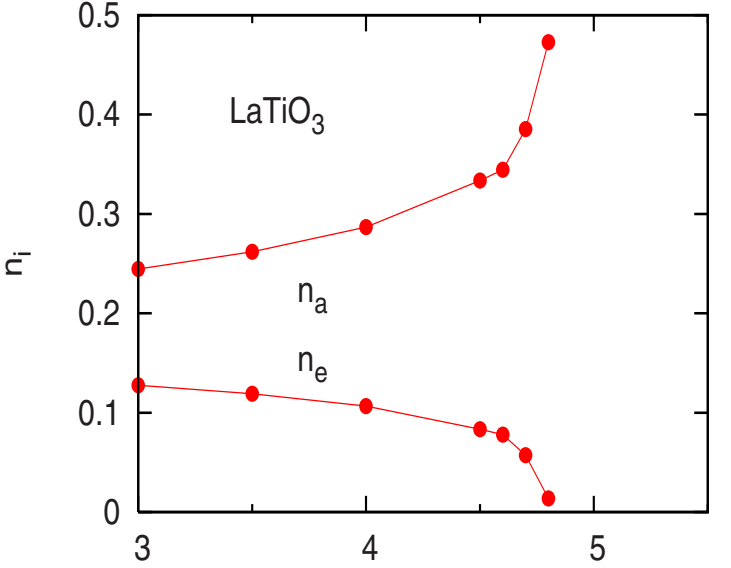

(a)

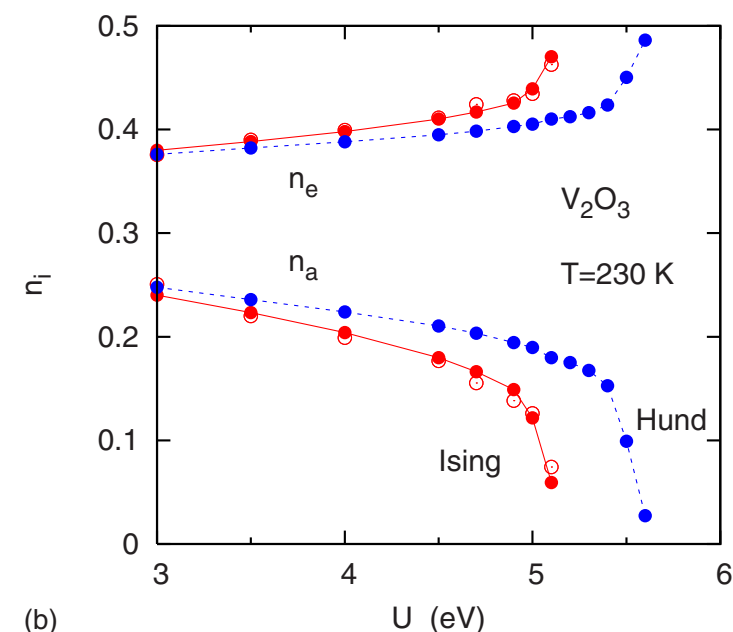

(b)

$\mathrm{U}(\mathrm{eV})$

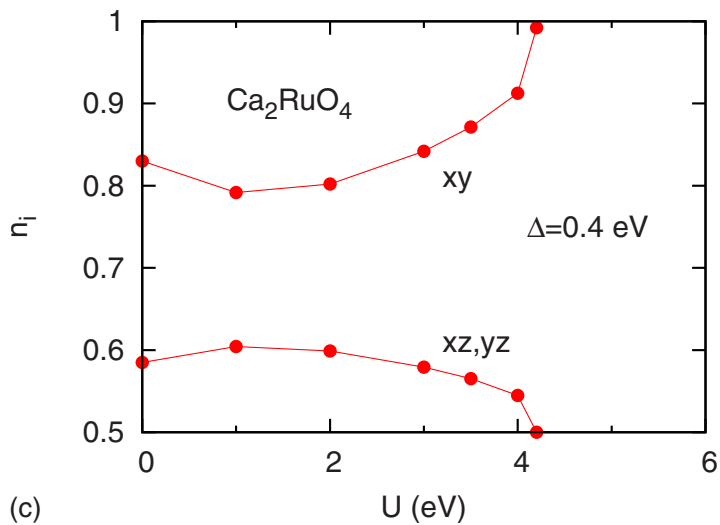

FIG. 9. (Color online) Subband occupancies obtained within finite-temperature multiband ED/DMFT as functions of $U$. Upper panels: $\mathrm{LaTiO}_{3}\left(3 d^{1}\right.$ configuration) for full Hund exchange with $J=0.65 \mathrm{eV}$ (Ref. 4); $\mathrm{V}_{2} \mathrm{O}_{3}\left(3 d^{2}\right.$ configuration) for full Hund exchange (blue dots) and Ising-like exchange (red solid dots) with $J=0.7 \mathrm{eV} ;{ }^{56}$ red empty dots: quantum Monte Carlo DMFT results (Ref. 5). Lower panel: $\mathrm{Ca}_{2} \mathrm{RuO}_{4}\left(4 d^{4}\right.$ configuration) for full Hund exchange with $J=U / 4$ and $\Delta=0.4 \mathrm{eV} t_{2 g}$ crystal-field splitting (Ref. 8).

while others do not does not appear appropriate. As shown consistently by all orbital-resolved spectra in the present multisite ED/DMFT study, there is a single Mott transition common to all cluster molecular orbitals, implying a simultaneous opening of the Mott gap across the entire Fermi surface. Nevertheless, in agreement with previous authors, we find pronounced momentum variation in quasiparticle properties close to the Mott transition.

In view of the approximate nature of the momentum variation in the lattice self-energy and Green's function derived within the CDMFT, it would be very interesting to compare the above results with analogous ones obtained within the DCA, ${ }^{20,36}$ in particular, since the cluster molecular-orbital components of the density of states, as stated above, differ appreciably between these two cluster DMFT schemes. ${ }^{59}$ This comparison will be addressed in a future publication.

The scenario discussed in this section differs strikingly from the one found for several multiorbital materials, which have been studied previously by various groups. For the sake of comparison we review some of these systems in the following section.

\section{COMPARISON WITH MULTIORBITAL SYSTEMS}

During the recent years single-site multiorbital DMFT has been used extensively to investigate the metal insulator transition of a variety of materials. ${ }^{1}$ Here, we briefly discuss some of these systems, which are regarded as typical Mott insulators, and which all exhibit characteristic changes of the electronic structure as the metallic phase is replaced by the insulator at large Coulomb energies.

Figure 9 shows the correlation-driven enhancement of orbital polarization for $\mathrm{LaTiO}_{3}, \mathrm{~V}_{2} \mathrm{O}_{3}$, and $\mathrm{Ca}_{2} \mathrm{RuO}_{4}$. Because of the orthorhombic structure of $\mathrm{LaTiO}_{3}, \mathrm{LDA}$ calculations reveal that the $a_{g}$ subbands of the $t_{2 g}$ sector are slightly more occupied than the two $e_{g}^{\prime}$ components. ${ }^{3}$ Local Coulomb interactions enhance this $t_{2 g}$ crystal-field splitting so that at the Mott transition close to $U=5 \mathrm{eV}$ the $e_{g}^{\prime}$ bands become nearly empty and the $a_{g}$ band half filled. ${ }^{3,4}$ In the case of $\mathrm{V}_{2} \mathrm{O}_{3}$, the corundum lattice structure ensures that the doublydegenerate $e_{g}^{\prime}$ bands have slightly larger binding energy than the $a_{g}$ bands. ${ }^{5}$ With increasing Coulomb interaction this crystal-field splitting is strongly enhanced until in the range $U \approx 5 \ldots 6 \mathrm{eV}$, the $a_{g}$ bands are pushed above the Fermi level, and the $e_{g}^{\prime}$ bands become half filled. ${ }^{5,6,56}$ Finally, in the 


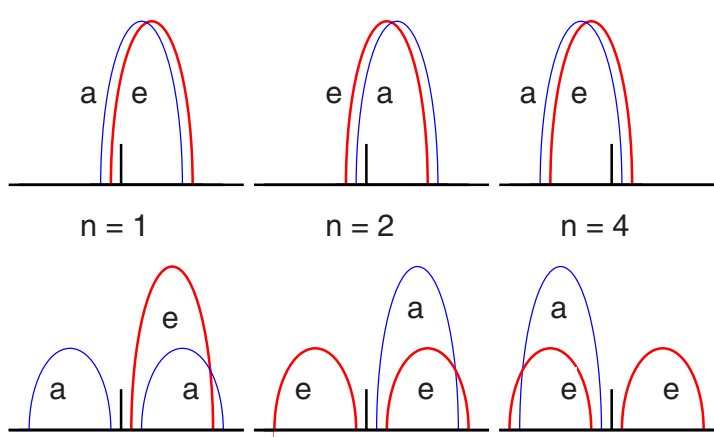

FIG. 10. (Color online) Schematic illustration of correlation driven enhancement of orbital polarization. Upper row: crystal-field split $t_{2 g}$ LDA densities of states for occupancies $n=1,2$, and 4 , corresponding to $\mathrm{LaTiO}_{3}, \mathrm{~V}_{2} \mathrm{O}_{3}$ and $\mathrm{Ca}_{2} \mathrm{RuO}_{4}$, respectively. Blue curves: singly-degenerate $a_{g}$ band, red curves: doubly-degenerate $e_{g}^{\prime}$ bands. In the case of $\mathrm{Ca}_{2} \mathrm{RuO}_{4}, a$ refers to $d_{x y}, e$ to $d_{x z, y z}$. The vertical bars denote the Fermi level. Lower row: orbitally polarized Mott phase. $n=1$ : empty $e_{g}^{\prime}$ bands, lower and upper Hubbard peaks of half-filled $a_{g}$ band; $n=2$ : empty $a_{g}$ band, lower and upper Hubbard peaks of half-filled $e_{g}^{\prime}$ bands; $n=4$ : filled $a_{g}$ band, lower and upper Hubbard peaks of half-filled $e_{g}^{\prime}$ bands.

case of $\mathrm{Ca}_{x} \mathrm{Sr}_{2-x} \mathrm{RuO}_{4}$, $\mathrm{Sr}$ substitution via the smaller $\mathrm{Ca}$ ions gives rise to an enlarged crystal-field splitting between $d_{x y^{-}}$ and $d_{x z, y z}$-like subbands. ${ }^{60}$ Coulomb correlations increase this splitting until in the Mott phase the $d_{x y}$ bands are fully occupied and the half-filled $d_{x z, y z}$ bands are split into lower and upper Hubbard bands. ${ }^{8}$

Schematically, the uncorrelated densities of states of these transition-metal oxides and the spectra derived within singlesite multiorbital DMFT for realistic Coulomb energies are shown in Fig. 10. Note that in the metallic phase, both orbital symmetries contribute to the spectral weight at the Fermi level. In the Mott phase, the gap involves transitions between states of opposite symmetry character.

Despite the different subband occupancies of these materials, they exhibit a similar correlation-driven enhancement of orbital polarization. ${ }^{61}$ By pushing some subbands above or below the Fermi level, the effective degeneracy is reduced from three to two or one. The Mott transition therefore occurs at lower critical Coulomb energy than in a cubic environment with equivalent subbands.

According to Fig. 1, the cluster molecular-orbital densities of the single-band Hubbard model in a multisite picture also exhibit a substantial splitting relative to the total band width. Nevertheless, Coulomb correlations in these cases only lead to moderate charge transfer between these cluster orbitals, as demonstrated by the results given in Fig. 2. The main physical reason for this qualitative difference with respect to the multiorbital materials is the strong single-particle hybridization among orbitals so that any tendency toward orbitalselective Mott transitions is suppressed. For the same reason, partial band filling or emptying, with a Mott transition in the remaining subset of bands, as found in several multiorbital materials, is also absent.

A certain amount of interorbital hybridization exists also in multiorbital systems since the single-electron Hamiltonian in the orbital basis is not diagonal throughout the Brillouin
Zone. This residual coupling, however, is much weaker than in the single-band multisite system so that Coulomb correlations can indeed lead to nearly complete orbital polarization. This is supported by the DMFT results for $\mathrm{LaTiO}_{3}$ and $\mathrm{V}_{2} \mathrm{O}_{3}$, which have been studied both by evaluating the lattice Green's function via Eq. (2) (see Refs. 3 and 6) and via the approximate version, Eq. (29) (see Refs. 4, 5, and 56). In these systems both formulations give very similar results, in particular, both confirm the scenario of strong orbital polarization.

\section{CONCLUSION}

Cellular DMFT combined with finite-temperature exact diagonalization has been used to investigate the influence of short-range correlations on the Mott transition in the singleband Hubbard model. Both square and triangular lattices at half filling were studied. A mixed basis consisting of cluster sites and bath molecular orbitals was shown to provide an efficient method for the evaluation of the cluster selfenergies and Green's functions. Since in the cluster molecular-orbital representation these quantities become diagonal, an intriguing analogy exists between Coulomb correlations in these multisite single-band systems and several multiorbital materials, which were studied previously within single-site DMFT.

In remarkable contrast to $\mathrm{LaTiO}_{3}, \mathrm{~V}_{2} \mathrm{O}_{3}$, and $\mathrm{Ca}_{2} \mathrm{RuO}_{4}$, which exhibit pronounced orbital polarization at the Mott transition, the single-band systems show very little correlation-driven enhancement of orbital polarization. Thus, all cluster molecular orbitals take part in the metal-insulator transition. Moreover, the transition occurs at the same critical $U$ for all cluster orbitals. Since an approximate momentum variation in the lattice self-energy and Green's function can be constructed from a superposition of these molecularorbital components, this finding yields the important result that the Mott gap opens simultaneously across the entire Fermi surface. Thus, for both square and triangular lattices at half filling, there is no orbital-selective Mott transition, where certain sections of the Brillouin Zone would open a gap at lower Coulomb energy than other parts. Moreover, there is no evidence for the combination of subband filling and Mott transition in other subbands that is characteristic of the multiorbital materials mentioned above. It would be of great interest to investigate whether these findings also hold at a finer momentum resolution, which would require cluster sizes larger than $n_{c}=4$.

\section{ACKNOWLEDGMENTS}

We like to thank A. Georges, N. Kawakami, G. Kotliar, R.H. McKenzie, M. Potthoff, B.J. Powell, and H. Tsunetsugu for useful discussions. The computational work was carried out on the Jülich JUMP computer. 
${ }^{1}$ For recent reviews, see K. Held, Adv. Phys. 56, 829 (2007); G. Kotliar, S. Y. Savrasov, K. Haule, V. S. Oudovenko, O. Parcollet, and C. A. Marianetti, Rev. Mod. Phys. 78, 865 (2006).

${ }^{2}$ A. Georges, G. Kotliar, W. Krauth, and M. J. Rozenberg, Rev. Mod. Phys. 68, 13 (1996).

${ }^{3}$ E. Pavarini, S. Biermann, A. Poteryaev, A. I. Lichtenstein, A. Georges, and O. K. Andersen, Phys. Rev. Lett. 92, 176403 (2004).

${ }^{4}$ A. Liebsch, Phys. Rev. B 77, 115115 (2008).

${ }^{5}$ G. Keller, K. Held, V. Eyert, D. Vollhardt, and V. I. Anisimov, Phys. Rev. B 70, 205116 (2004).

${ }^{6}$ A. I. Poteryaev, J. M. Tomczak, S. Biermann, A. Georges, A. I. Lichtenstein, A. N. Rubtsov, T. Saha-Dasgupta, and O. K. Andersen, Phys. Rev. B 76, 085127 (2007).

${ }^{7}$ V. I. Anisimov, I. A. Nekrasov, D. E. Kondakov, T. M. Rice, and M. Sigrist, Eur. Phys. J. B 25, 191 (2002)

${ }^{8}$ A. Liebsch and H. Ishida, Phys. Rev. Lett. 98, 216403 (2007).

${ }^{9}$ F. Lechermann, S. Biermann, and A. Georges, Phys. Rev. Lett. 94, 166402 (2005).

${ }^{10}$ M. De Raychaudhury, E. Pavarini, and O. K. Andersen, Phys. Rev. Lett. 99, 126402 (2007).

${ }^{11}$ H. Ishida and A. Liebsch, Phys. Rev. B 77, 115350 (2008).

${ }^{12}$ A. Koga, N. Kawakami, T. M. Rice, and M. Sigrist, Phys. Rev. Lett. 92, 216402 (2004); Physica B 359-361, 1366 (2005).

${ }^{13}$ A. Liebsch, Phys. Rev. B 70, 165103 (2004); Phys. Rev. Lett. 95, 116402 (2005).

${ }^{14}$ Yun Song and L.-J. Zou, Phys. Rev. B 72, 085114 (2005).

${ }^{15}$ K. Inaba, A. Koga, S. Suga, and N. Kawakami, J. Phys. Soc. Jpn. 74, 2393 (2005).

${ }^{16}$ T. A. Costi and A. Liebsch, Phys. Rev. Lett. 99, 236404 (2007).

${ }^{17}$ T. Tohyama and S. Maekawa, Phys. Rev. B 49, 3596 (1994).

${ }^{18}$ R. Preuss, W. Hanke, and W. von der Linden, Phys. Rev. Lett. 75, 1344 (1995).

${ }^{19}$ A. Moreo, S. Haas, A. W. Sandvik, and E. Dagotto, Phys. Rev. B 51, 12045 (1995).

${ }^{20}$ M. H. Hettler, A. N. Tahvildar-Zadeh, M. Jarrell, T. Pruschke, and H. R. Krishnamurthy, Phys. Rev. B 58, R7475 (1998).

${ }^{21}$ D. Senechal, D. Perez, and M. Pioro-Ladriere, Phys. Rev. Lett. 84, 522 (2000).

${ }^{22}$ A. I. Lichtenstein and M. I. Katsnelson, Phys. Rev. B 62, R9283 (2000).

${ }^{23}$ C. Huscroft, M. Jarrell, Th. Maier, S. Moukouri, and A. N. Tahvildarzadeh, Phys. Rev. Lett. 86, 139 (2001).

${ }^{24}$ S. Moukouri and M. Jarrell, Phys. Rev. Lett. 87, 167010 (2001).

${ }^{25}$ G. Kotliar, S. Y. Savrasov, G. Palsson, and G. Biroli, Phys. Rev. Lett. 87, 186401 (2001).

${ }^{26}$ Y. Imai and N. Kawakami, Phys. Rev. B 65, 233103 (2002).

${ }^{27}$ Th. A. Maier, Th. Pruschke, and M. Jarrell, Phys. Rev. B 66, 075102 (2002).

${ }^{28}$ S. Onoda and M. Imada, Phys. Rev. B 67, 161102(R) (2003).

${ }^{29}$ B. Kyung, J. S. Landry, D. Poulin, and A.-M. S. Tremblay, Phys. Rev. Lett. 90, 099702 (2003).

${ }^{30}$ M. Potthoff, M. Aichhorn, and C. Dahnken, Phys. Rev. Lett. 91, 206402 (2003).
${ }^{31}$ D. Senechal and A.-M. S. Tremblay, Phys. Rev. Lett. 92, 126401 (2004).

${ }^{32}$ O. Parcollet, G. Biroli, and G. Kotliar, Phys. Rev. Lett. 92, 226402 (2004).

${ }^{33}$ M. Capone, M. Civelli, S. S. Kancharla, C. Castellani, and G. Kotliar, Phys. Rev. B 69, 195105 (2004).

${ }^{34}$ D. Senechal, P.-L. Lavertu, M.-A. Marois, and A.-M. S. Tremblay, Phys. Rev. Lett. 94, 156404 (2005).

${ }^{35}$ M. Civelli, M. Capone, S. S. Kancharla, O. Parcollet, and G. Kotliar, Phys. Rev. Lett. 95, 106402 (2005).

${ }^{36}$ T. Maier, M. Jarrell, T. Pruschke, and M. H. Hettler, Rev. Mod. Phys. 77, 1027 (2005).

${ }^{37}$ B. Kyung, S. S. Kancharla, D. Senechal, A.-M. S. Tremblay, M. Civelli, and G. Kotliar, Phys. Rev. B 73, 165114 (2006).

${ }^{38}$ M. Capone and G. Kotliar, Phys. Rev. B 74, 054513 (2006).

${ }^{39}$ B. Kyung, Phys. Rev. B 75, 033102 (2007).

${ }^{40}$ J. Merino, Phys. Rev. Lett. 99, 036404 (2007).

${ }^{41}$ Y. Z. Zhang and M. Imada, Phys. Rev. B 76, 045108 (2007).

${ }^{42}$ T. Ohashi, T. Momoi, H. Tsunetsugu, and N. Kawakami, Phys. Rev. Lett. 100, 076402 (2008).

${ }^{43}$ P. Sahebsara and D. Senechal, Phys. Rev. Lett. 100, 136402 (2008).

${ }^{44}$ H. Park, K. Haule, and G. Kotliar, arXiv:0803.1324 (unpublished).

${ }^{45}$ E. Koch, G. Sangiovanni, and O. Gunnarsson, Phys. Rev. B 78, 115102 (2008).

${ }^{46}$ E. Gull, Ph. Werner, M. Troyer, and A. J. Millis, arXiv:0805.3778 (unpublished).

${ }^{47}$ M. Ferrero, P. S. Cornaglia, L. De Leo, O. Parcollet, G. Kotliar, and A. Georges, arXiv:0806.4383 (unpublished).

${ }^{48} \mathrm{H}$. Lee, G. Li, and H. Monien, arXiv:0807.1683 (unpublished).

${ }^{49}$ M. Balzer and M. Potthoff, arXiv:0808.2364 (unpublished).

${ }^{50} \mathrm{An}$ excellent introduction to quantum cluster models can be found in: D. Senechal, arXiv:0806.2690 (unpublished).

${ }^{51}$ See also: M. Ferrero, L. De Leo, Ph. Lecheminant, and M. Fabrizio, J. Phys.: Condens. Matter 19, 433201 (2007).

${ }^{52}$ M. Caffarel and W. Krauth, Phys. Rev. Lett. 72, 1545 (1994).

${ }^{53}$ C. A. Perroni, H. Ishida, and A. Liebsch, Phys. Rev. B 75, 045125 (2007). See also A. Liebsch and T. A. Costi, Eur. Phys. J. B 51, 523 (2006).

${ }^{54}$ R. B. Lehoucq, D. C. Sorensen, and C. Yang, ARPACK Users' Guide (SIAM, Philadelphia, 1997).

${ }^{55}$ A. Liebsch and H. Ishida, Eur. Phys. J. B 61, 405 (2008).

${ }^{56}$ A. Liebsch (unpublished).

${ }^{57}$ See also: M. Capone, L. de Medici, and A. Georges, Phys. Rev. B 76, 245116 (2007).

${ }^{58}$ The fact that differences between Eqs. (2) and (29) can be important was also emphasized by Poteryaev et al. (Ref. 6).

${ }^{59}$ For the square lattice with $n_{c}=4$, see, e.g., K. Haule and G. Kotliar, Phys. Rev. B 76, 104509 (2007).

${ }^{60}$ Z. Fang, N. Nagaosa, and K. Terakura, Phys. Rev. B 69, 045116 (2004).

${ }^{61}$ Note, however, other trends such as those discussed in Refs. 9-11. 